\title{
Los tisanópteros del grupo genérico Anaphothrips (Thysanoptera: Thripidae), con énfasis en América Central
}

\author{
Axel P. Retana-Salazar ${ }^{1,2}$ \\ 1 Centro de Investigación en Estructuras Microscópicas (CIEMIC). Ciudad de la Investigación, Universidad de Costa \\ Rica, 2060. \\ 2 Instituto de Investigación en Biología y Conservación, San Pedro de Montes de Oca, San José, Costa Rica; apretana@ \\ cariari.ucr.ac.cr
}

Recibido 02-IX-2004. Corregido 10-X-2006. Aceptado 24-X-2006.

\begin{abstract}
Thysanopterans of the generic group Anaphothrips (Thripidae: Thripinae) with emphasis in Central America. A taxonomic revision and phylogenetic analysis of the generic group Anaphothrips are presented. Several genera in Central America are closely related to this generic group. Based on the analysis of characters and its possible evolution, the new genus Nakaharathrips has been segregated from Anaphothrips, and the taxonomic status of the subspecies of Aurantothrips has been changed to the species level. The phylogenetic analysis shows a close relationship between the genera Anaphothrips, Ranjana, Nakaharathrips n.gen., Aurantothrips, Nicolemma n.gen., Ameranathrips, Baileyothrips and Gonzalezya n.gen., where the first lineage is formed by the genera (Ranjana (Aurantothrips + Nicolemma)) and the second lineage contains the genera [(Anaphothrips + Nakaharathrips)(Baileyothrips (Ameranathrips + Gonzalezya)]. Rev. Biol. Trop. 55 (1): 321333. Epub 2007 March. 31.
\end{abstract}

Key words: Anaphothrips group, new status, phylogenetics, new genus, evolution of characters.

Dentro del orden Thysanoptera, el grupo genérico Anaphothrips ha sido estudiado por Bhatti (1978) en lo correspondiente a la Región Oriental, donde elevó los subgéneros al nivel de género. Al mismo tiempo describe un nuevo género para una especie de difícil ubicación (Bhatti 1978).

El parentesco entre los géneros cercanos no es claro a pesar de los caracteres morfológicos compartidos. Mound y Marullo (1996) manifiestan que la forma costarricense de Aurantothrips orchidiaceus presenta siete segmentos en la antena, a diferencia de otras regiones donde presenta ocho segmentos; sin embargo, un estudio a fondo de la morfología de estos especímenes indica que esto no parece tener asidero.

Por su parte Ameranathrips y Baileyothrips tienen variaciones que deben ser tomadas en cuenta al estudiar el grupo en el área centroamericana. Nakahara (1995) en su revisión del género Anaphothrips para América del Norte reduce el número de especies. Para este autor el centro de origen de este género es en el norte debido a su poca abundancia en las regiones tropicales. Uno de los pocos registros que se tiene de este grupo en el neotrópico es la especie flavocastaneus descrita por Johansen (1981) para México y que fue transferida al género Oxithrips por Nakahara (1995).

Entre las características importantes que manifiesta Anaphothrips está la presencia de especies con ocho segmentos en la antena y otras con nueve; esto se debe a la división del segmento VI de la antena, dando origen a un nuevo segmento. Este tipo de novedades evolutivas son comunes entre los géneros emparentados con Anaphothrips y de ahí que merece particular atención este grupo. 


\section{CARACTERÍSTICAS GENERALES DE LOS GÉNEROS EMPARENTADOS CON Anaphothrips EN CENTROAMÉRICA}

Coloración café pálido excepto en los géneros Aurantothrips (2 especies) y Nicolemma n.gen. (1 especie). Habitualmente con las setas mayores del pronoto reducidas, con ornamentación poco evidente y de pocas a muchas setas discales del pronoto.

Inusualmente se presentan géneros con solo siete segmentos en la antena (Nicolemma n.gen.). La mayor parte presentan ocho antenómeros: Anaphothrips en parte, Ameranathrips, Gonzalezya n.gen. y Baileyothrips. Presentan nueve segmentos un grupo de especies de Anaphothrips entre las que se cuenta la especie tipo del género. Presentan ojos bien desarrollados y sin facetas pigmentadas en su mayoría; cuatro pares de setas en la parte ventral de la cabeza entre el vertex y la parte anterior del cono bucal.

Setas discales del meso y metanoto bien desarrolladas, ornamentación del mesonoto $\mathrm{y}$ el metanoto fuerte $\mathrm{y}$ evidente constituida por líneas paralelas. En Gonzalezya n.gen. se evidencian un par de setas posteroangulares bien desarrolladas (autoapomorfía del género). Hileras de setas del ala I habitualmente continuas o con espacios muy reducidos, entre ellas en los géneros Aurantothrips y Nicolemma n.gen.

Habitualmente no presentan espermateca desarrollada, excepto en la única especie del género Nicolemma n.gen., que presenta una espermateca bien desarrollada, esclerotizada y esférica.

Desde el punto de vista biológico los géneros Aurantothrips, Ameranathrips, Nicolemma n.gen. y Gonzalezya n.gen. se hallan asociados a flores, mientras que los géneros Baileyothrips, Anaphothrips sensu stricto y Anaphothrips (nuevo estatus) se hallan asociados a zacates (Poaceae). Esto en realidad señala linajes separados en función de sus caracteres (RetanaSalazar y Retana-Salazar, 2004).

\section{Consideraciones taxonómicas}

El género Anaphothrips fue descrito por Uzel en 1895 a partir de la especie Thrips obscura descrita por Müller. Cerca de 80 especies fueron descritas para este género en varios subgéneros (Jacot-Guillarmod 1974), informados para todos los continentes excepto la Antártida.

Anaphothrips se consideraba un grupo parafilético hasta que Bhatti (1978) revisó el grupo y elevó a género a los subgéneros: Agalmothrips, Anaphothrips, Dictyothrips, Hyalopterothrips, Proscirtothrips y Tamaricothrips, igualmente sinonimizó el subgénero Neophysopus con Anaphothrips. Traslada dos especies al género Tusothrips y describe el género Ranjana para la especie cingulatus, la cual había estado ubicada inicialmente en Euthrips, luego en Anaphothrips y por último en Scirtothrips.

La situación del género Anaphothrips ha sido parcialmente resuelta por los estudios de Bhatti (1978) y Nakahara (1995), sin embargo aun quedan fuertes dudas acerca de las relaciones entre los géneros del Nuevo Mundo emparentados con este género. En este trabajo se describen dos nuevos géneros emparentados con Anaphothrips, el género Nicolemma n.gen. que presenta siete segmentos en la antena y una espermateca bien desarrollada y el género Gonzalezya n.gen. con la especie marinae asociada a flores de anturio.

Por último, se trata desde un punto de vista evolutivo la presencia de ocho o nueve antenómeros en las especies del género Anaphothrips, $\mathrm{y}$ en función de la resolución filogenética y de la consistencia estructural de los grupos (Retana-Salazar y Retana-Salazar, en prensa) se propone la separación del género Anaphothrips caracterizado por la presencia de nueve antenómeros y de Nakaharathrips n.gen. para el grupo de especies de ocho segmentos en la antena.

\section{Análisis filogenético}

El grupo Anaphothrips es particularmente complicado debido a que la separación de los 
géneros y las especies se ha efectuado tradicionalmente utilizando caracteres que fácilmente convergen en linajes muy diferentes. Esto ha obligado a agrupar especies de forma irregular y por consiguiente el grupo es difuso en cuanto a su consistencia (Retana-Salazar y RetanaSalazar, en prensa).

En el presente trabajo se utilizaron 24 caracteres morfológicos (apéndice A) descritos en la literatura por Nakahara (1995) y Bhatti (1978) otros han sido utilizados por Palmer y Mound (1985), Mound et al. (1980) y otros han sido estudiados por el autor. Con la finalidad de darle polaridad a los caracteres se utilizó la técnica del out-group y se escogió para este fin el género Oxithrips, se complementó este resultado con el uso de un ancestro hipotético deducido a partir de la observación de los caracteres en los géneros tanto centroamericanos como del Viejo Mundo. Se obtuvieron dos arreglos filogenéticos igualmente parsimoniosos según los programas TREEGARDENER y PAUP 3.1.1, el consenso estricto muestra una politomía entre los grupos (Baileyothrips + (Anaphoth rips + Nakaharathrips $)+($ Ameranathrips + Gonza lezya n.gen.)) de los táxones estudiados.
Posteriormente se utilizó el método de Brooks y Wiley (1986) para medida de la entropía evolutiva asociada a la topología, con lo que se obtuvo el arreglo filogenético más estable y con mayor información evolutiva de los caracteres (Fig. 9, Apéndice 1).

(()Ranjana $+($ Aurantothrips + Nicolemma n.gen. $))+(($ Anaphothrips + Nakaharathrips n.g en. $)+(($ Baileyothrips $+($ Ameranathrips + Gonza lezya n.gen.)))) esta topología muestra los siguientes valores de sus estadísticos filogenéticos $\mathrm{ci}=0.75, \mathrm{~L}=41, \mathrm{ri}=0.7$.

El grupo se divide en dos grandes linajes el primero es el grupo Ranjana constituido por este género, y su grupo hermano constituido por Aurantothrips y Nicolemma n.gen. (Cuadro 1).

Un segundo linaje está constituido por los géneros Anaphothrips, Nakaharathrips n.gen., Baileyothrips, Ameranathrips y Gonzalezya n.gen., distinguiéndose dos agrupamientos uno con los géneros Anaphothrips y Nakaharathrips n.gen., otro conformado por los géneros Baileyothrips, Ameranathrips y Gonzalezya n.gen. donde se evidencia que Baileyothrips es el grupo basal y su adelfotaxon está formado por Ameranathrips y Gonzalezya n.gen. (Cuadro 2).

\section{Clave para la determinación de los géneros del grupo Anaphothrips con énfasis en los táxones de América Central}

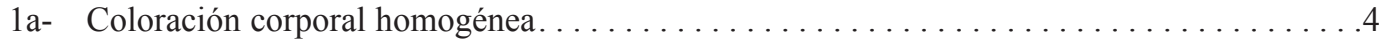

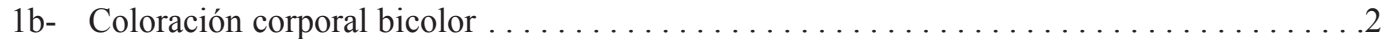

2a- Cuerpo bicolor, lateralmente amarillo con una banda longitudinal café, setas posteroangulares reducidas y subiguales a las setas discales del pronoto, solo espínula mesotorácica presente

2b- Cuerpo bicolor en sentido anteroposterior (cabeza y pterotórax café oscuro, resto del cuerpo amarillo claro), setas posteroangulares reducidas, espínulas en meso y metaesterno

Ranjana Bhatti

3a- Espermateca ausente, con 8 antenómeros............... Aurantothrips Bhatti

3b- Espermateca presente, con 7 antenómeros .............. Nicolemma n.gen.

4a- Con craspedum presentes en los segmentos abdominales $\ldots \ldots \ldots \ldots \ldots \ldots \ldots \ldots \ldots$

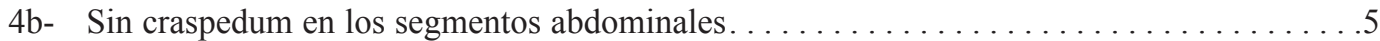


5a- Con 9 antenómeros o al menos una sutura parcial que indica una división del antenómero

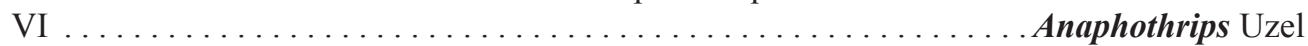

5b- Con 8 antenómeos sin evidencias de pseudosegmentación de la antena .Nakaharathrips n.gen.

6a- Setas sobre las venas del ala I discontinuas .......... Ameranathrips Mound y Marullo

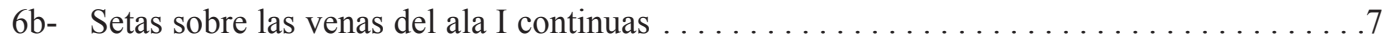

7a- Setas posteroangulares no desarrolladas, tres pares de setas ocelares $\ldots \ldots \ldots \ldots \ldots \ldots \ldots \ldots \ldots \ldots \ldots \ldots \ldots \ldots \ldots \ldots \ldots \ldots \ldots \ldots \ldots \ldots \ldots$ Baileyothrips Kono y O’Neill

7b- Setas posteroangulares desarrolladas (1/4 la longitud del eje anteroposterior del tórax) Gonzalezya n.gen.

\section{Diagnosis del género Ranjana Bhatti}

Presenta espínula meso y metatorácica, reducción de la quetotaxia del pronoto, tergos abdominales sin microtrichias, pronoto con esculturación poco profusa, setas discales pequeñas y sin arreglo en líneas definidas.

\section{Descripción de nuevos géneros y cambios taxonómicos propuestos}

Nicolemma gen.nov.

Especie tipo: Nicolemma garitai n.sp. por designación original (Fig 1-6, 7D)

English diagnosis: This genus is easy to distinguish because of its antenae with seven segments, and a sclerotized and spheric spermaeteca in abdominal segments VII-VIII

Descripción: Cabeza con tres pares de setas ocelares, con una serie de pequeñas setas posoculares, antena con siete antenómeros (Fig. 7D), con sensoria bifucados en III-IV. Pronoto con pocas setas todas reducidas y subiguales a las setas discales, seta pm 1 más desarrollada que las demás. Setas externas del metanoto en el margen anterior, setas internas del metanoto en el medio del esclerito. Vena 1 con una hilera de setas incompleta, vena II con una hilera de setas completa, tégula con 6-8 setas. Tergitos y esternitos sin craspeda, setas tergales medias alejadas entre sí, tergito VIII con peine posteromarginal completo, de microtrichias largas y muy cercanas entre sí. El caracter diagnóstico principal es la presencia de una espermateca esférica bien desarrollada, visible a la altura de los tegitos VII-VIII. Macho desconocido.

Etimología: Este género ha sido nombrado como homenaje a Nicole y Emma

Comentario: La primera vez que esta especie fue recolectada fue en 1991 en el Jardín Botánico Lankester, en hojas de Encyclia, pocos especímenes fueron rerecolectados en ese momento. Mound y Marullo (1996) postulan que esta variación morfológica en el número de antenómeros no es un buen carácter para la separación de especies. Con mayor número de especímenes se pudo determinar que aparte del número de antenómeros estos especímenes cuentan con una espermateca esférica entre los tergitos VII-VIII. Este conjunto de caracteres es único en el orden y justifican la separación de un nuevo género.

\section{Nicolemma garitai n.sp. (Fig. 1-6)}

English diagnosis: similar to the genus; forewing with complete row of setae little separated by spaces; basal formula: setae 10 , medial setae 2 and apical setae 7.

Material: Holotipo (hembra). Costa Rica recolectada en: Cartago. En el jardín botánico Lankaster, en flores de Encyclia fragans. 13-I91 (ARS 1191). Dos hembras recolectadas en el Jardín Botánico Wilson en varias especies de orquídea. Depositado en la colección de Axel P. Retana-Salazar (en el MIUCR). Paratipos: tres hembras recolectadas con el holotipo y 


\section{CUADRO 1}

Características que justifican el linaje Ranjana, constituido por tres géneros y cuatro especies

Caracteres/ táxones

3) Patrón de coloración corporal

9) Setas del ala I

10-2) Distribución de las setas del ala I

20) Seta medial del segmento IX del abdomen

7) Forma de la cabeza

21) Setas mediales del segmento IX del abdomen modificadas

\section{Ranjana}

Bicolor

Discontinuas

Dispuestas con al menos un claro evidente entre la hilera continua de setas

Presente
Aurantothrips

Bicolor

Discontinuas

Dispuestas con al menos un claro evidente entre la hilera continua de setas

Presente

Mayor en su diámetro anteroposterior que en su diámetro transversal
Nicolemma n. gen.

Bicolor

Discontinuas

Dispuestas con al menos un claro evidente entre la hilera continua de setas

Presente

Mayor en su diámetro anteroposterior que en su diámetro transversal
Espiniformes
Espiniformes

CUADRO 2

Características que justifican el linaje Anaphothrips, constituido por cinco géneros y más de 50 especies

$\begin{array}{lll}\text { Caracteres/táxones } & \text { Anaphothrips } & \begin{array}{l}\text { Nakaharath } \\ \text { n. gen. }\end{array} \\ \begin{array}{l}\text { 8) Ommatidios de } \\ \text { colores presentes }\end{array} & \text { Presentes } & \text { Presentes } \\ \text { 6) Desarrollo de } & \text { Reducidos } & \text { Reducidos } \\ \text { los ocelos } & \text { Reducidas } \\ \text { 12) Setas discales } & \text { Reducidas } & \\ \text { del pronoto } & & \\ \text { 18) Craspeda } & & \\ \text { 19) Desarrollo de } & & \\ \text { los pleurotergitos } & & \\ \text { 20) Setas mediales } & & \\ \text { del segmento IX } & & \end{array}$

22) Espermateca

23) Textura de la espermateca

$\begin{array}{ll}\text { Presentes } & \text { Presentes } \\ \text { Reducidos } & \\ & \text { Presentes } \\ & \text { (convergente con } \\ & \text { linaje Ranjana, } \\ \text { debe considerarse } & \text { como un carácter } \\ \text { aparte) } & \text { Presente (converge } \\ \text { con Nicolemma, } \\ \text { debe considerarse } \\ \text { un carácter } \\ \text { separado) }\end{array}$

Presentes

Reducidos

Presentes (convergente con linaje Ranjana, debe considerarse como un carácter aparte)

Presente (converge con Nicolemma, debe considerarse un carácter separado)

Membranosa 

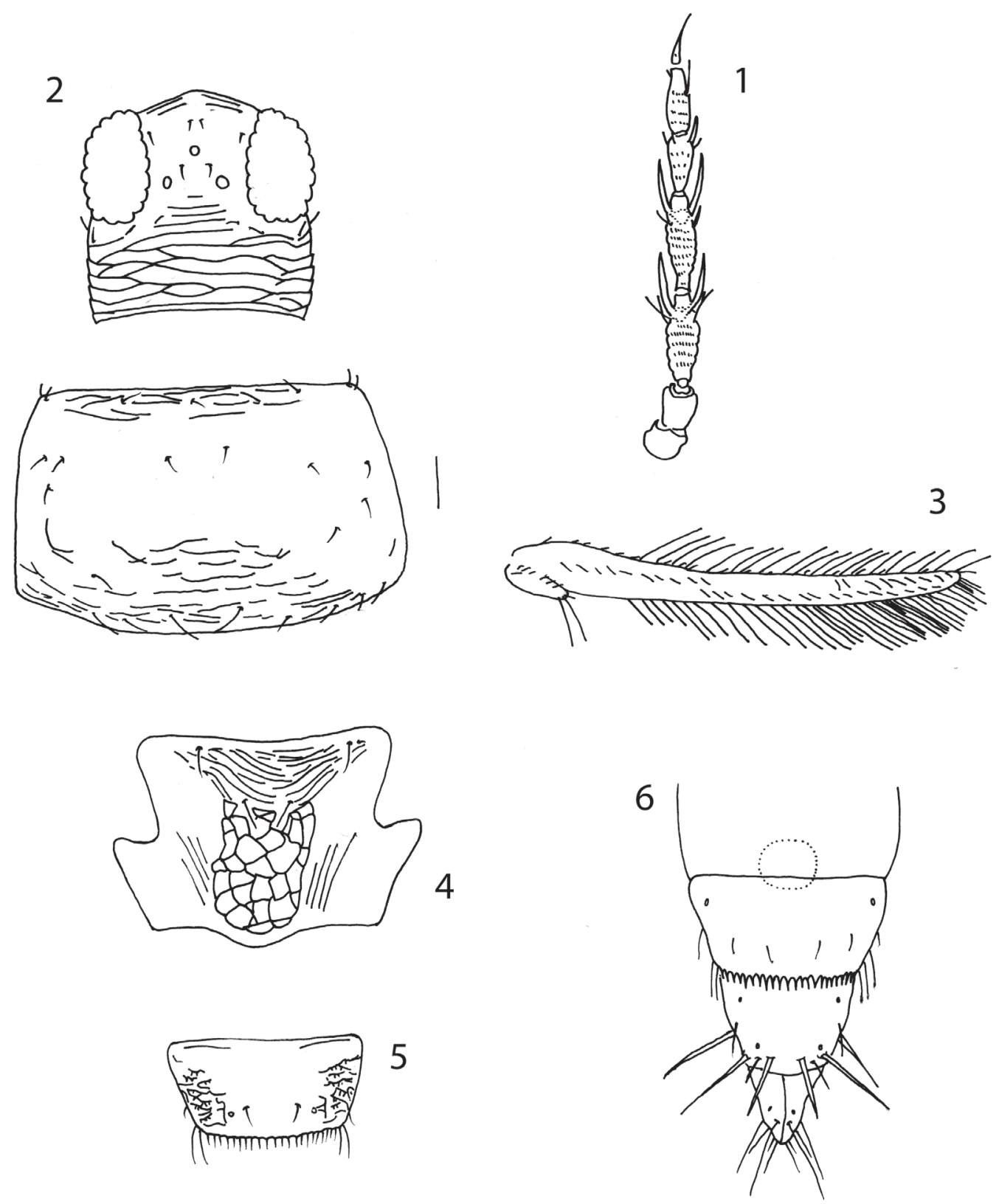

Fig. 1-6. Nicolemma n.gen garitai n.sp. Fig. 1. Antena. Fig. 2. Cabeza y pronoto. Fig. 3. Ala 1. Fig. 4. Metanoto. Fig. 5. Tergito VIII. Fig. 6. Sección entre los tergitos VII-VIII mostrando la espermateca esférica. 
dos hembras recolectadas en el Jardín Botánico Wilson. Paratipos depositados en Instituto de Biología de la Universidad Nacional Autónoma de México (IBUNAM), Senckenberg Museum of Frankfurt, Alemania (SMF) y Colección de Axel P. Retana-Salazar (en el MIUCR).

Hembra macróptera: Color (después de la maceración) amarillo pálido con una banda media longitudinal de color café oscuro, excepto en los segmentos IX-X; marcas oscuras en los tergitos nunca tocan el margen posterior. Cabeza amarillo pálido con marcas oscuras detrás de los ojos. Antena café oscuro, segmentos III-IV con el $1 / 5$ terminal amarillo pálido, segmento $\mathrm{V}$ con el $1 / 5$ basal amarillo pálido. Patas amarillo pálido. Alas anteriores café oscuro, con el cuarto basal pálido en el margen anterior, escama (tégula) café oscuro con el extremo proximal pálido. Alas posteriores pálidas con una línea central café oscuro. Microtrichia del ala de color café oscuro. Esculturación de la cabeza con líneas fuertes (Fig. 2), con una pequeña proyección del vertex, ocelos en una prominencia, ojos bien desarrollados, con cinco setas posoculares, poI ampliamente separada de la poII, cono bucal largo, proyectándose más allá de la mitad del protórax, antena con siete antenómeros, antenómeros III-IV con sensores bifurcados largos (Fig. 1). Pronoto y cabeza subiguales en longitud, todas las setas reducidas y subiguales en longitud, seta pmI el doble de larga de las setas discales, esculturación con fuertes líneas transversales. Alas y tégula (=escama) cubiertas con muchas microtrichias pequeñas. Ala anterior con hilera de setas en la vena 1 incompleta (10 setas basales, 2 setas mediales, 7 setas apicales) (Fig. 3), vena 2 sin setas en el cuarto basal, hilera de setas completa (20 setas), margen posterior con cilios ondulados, tégula con 7-8 setas y dos setas ciliares apicales con un esclerito en la cara ventral de la tégula. Ala posterior sin venas. Mesonoto con tres pares de setas marginales y un par de setas mediales, dos poros en el extremo apical, esculturación con fuertes líneas transversales y reticulación central. Metanoto con reticulación fuerte, par exteno de setas en el borde anterior, par interno de setas mediales (Fig. 4). Tergitos abdominales con setas mediales pequeñas, distancia entre las bases más de dos veces la longitud de la seta, segmento VIII con un peine completo de microtrichias (Fig. 5). Ovipositor débil con numerosos dientes pequeños. Espermateca esférica y traslúcida visible entre los segmentos VII-VIII del abdomen (Fig. 6).

Medidas (holotipo en $\mu \mathrm{m}$ ): Longitud total (distendido) 1698.4. Longitud de la cabeza 137.5, ancho 162.5; longitud vertex-cono bucal 312. Antenómro III longitud 72; sensor III longitud 56. Antenómero IV longitud 76; sensor IV longitud 56. Longitud del Pronoto 125, ancho 187.5; longitud setas discales 12.5; setas pmI 20-22.5.

Comentario: Morfológicamente cercano a Aurantothrips orchidaceus pero fácil de distinguir por los siete antenómeros y la espermateca esférica y esclerotizada. Se le halla en flores de Encyclia fragans en el Jardín Botánico Lankaster y en varias flores de orquídeas en el Jardín Botánico Wilson. Esta especie se halla sobre los pétalos de Encyclia usualmente en las manchas oscuras de las flores durante las horas calientes de la mañana. Mound y Marullo (1996) informan a orchidaceus en el follaje.

Etimología: dedicada a Jerson Garita.

Aurantothrips Bhatti, 1978: 90.

Type species: Anaphothrips orchidaceus Bagnall

Especie tipo: Anaphothrips orchidaceus Bagnall

Diagnosis: Cabeza con tres pares de setas ocelares, con una serie de pequeñas setas posoculares, antena con 8 segmentos (Fig. 7C), con sensores bifurcados en III-IV. Quetotaxia del pronoto reducida con pocas setas subiguales a las setas discales, seta pmI más desarrollada que las demás. Metanoto con par externo a nivel del margen anterior, setas internas en la parte medial del esclerito. Vena 1 con una hilera de incompleta de setas, vena II completa, tégula (=escama) con 6-8 setas. Tergitos y esternitos sin craspeda, setas mediotergales lejanas entre sí, terito VIII con peine completo 

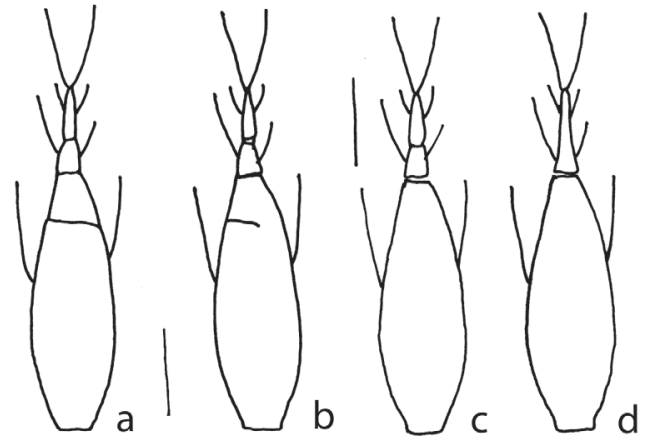

Fig. 7. Modelos de antenas en el grupo Anaphothrips (se ha simplificado al no dibujar las setas y los sensores). A-B. Anaphothrips sensu stricto. C. Nakaharathrips n.gen. D. Nicolemma n.gen.

de microtrichias largas. Macho (cuando se conoce) con dos pares de setas fuertes en el tergito IX, eternitos II-IV con áreas glandulares mediales.

Comentario: Mound y Marullo (1996) siguen el criterio de Sakimura (1967) y consideran los dos diferentes morfotipos como subespecies. No está claro si en el material rerecolectado hay una o más especies. Sakimura (1967) examinó 24 hembras, 9 machos, de este género, y concluye que en la muestra están presentes dos táxones, los cuales describe como subespecies con los nombres $A$. orchidaceus orchidaceus y A. ochidaceus orchidearum. Sin embargo, si tenemos un grupo difícil con un patrón de evolución complejo, y con una pobre definición de los caracteres con peso taxonómico y fiogenético para la determinación del nivel de especie, es fácil para los especialistas en el grupo mantener estos táxones como unidades diferentes, ya que esto facilita el estudio de los grupos de especies y el contenido de información biológica crece de una forma más rápida. En este trabajo se concluye que los caracteres externos son altamente conservados, particularmente el patrón de color, por lo que cualquier pequeña variación de estos puede señalar un cambio complementario en la biología del grupo que la exhibe. Este hecho puede ser suficiente para la separación de estas morfologías como especies separadas. Sin embargo, recientemente (Retana-Salazar y Retana-Salazar, en prensa) se ha propuesto una nueva metodología de separación de táxones, para lo cual debe cumplirse la relación c-inclusión de los caracteres, y se separa todo aquello que tenga un carácter o combinación de ellos que sea novedosa al plan básico descrito y definido para el taxon inmediato superior.

\section{Aurantothrips orchidaceus}

Anaphothrips orchidaceus

Bagnall, 1909: 33

Anaphothrips orchidearum

Bondar, 1931b: 435

Anaphothrips orchidaceus orchidaceus

Sakimura, 1967: 89-97

Anaphothrips orchidaceus orchidearum

Sakimura, 1967: 89-97

Aurantothrips orchidaceus

Bhatti, 1978: 90

Aurantothrips orchidaceus orchidaceus

Mound \& Marullo, 1996: 96

Aurantothrips orchidaceus orchidearum

Mound \& Marullo, 1996: 96

Diagnosis: Ala anterior con la tégula pálida al menos en la base, pero donde se une a la base del ala sombreado cerca del margen posterior, ápice del antenómero IV pálido, tergito IX del macho con un par de finas setas laterales a las setas dimórficas, antenómero $\mathrm{V}$ de la hembra 2.5-2.8 veces más largo que ancho, y 0.78-0.90 veces la longitud del antenómero VI, antenómero $\mathrm{V}$ del macho 2.3-2.5 veces más largo que ancho, y $0.75-0.83$ veces la longitud del antenómero VI.

Aurantothrips orchidearum n. stat.

Anaphothrips orchidearum

Bondar, 1931b: 435

Anaphothrips orchidaceus orchidearum

Sakimura, 1967: 89-97

Aurantothrips orchidaceus orchidearum

Mound \& Marullo, 1996: 96

Diagnosis: Ala 1 con la tégula o escama uniformente oscura, pero cerca de la base del ala es pálida, ápice del antenómero IV oscuro, tergito IX del macho con un par de setas finas 
entre las setas posteriores dimórficas, antenómero $\mathrm{V}$ de la hembra 2.1-2.2 veces más largo que ancho, y 0.7-0.74 veces la longitud del antenómero $\mathrm{VI}$, antenómero $\mathrm{V}$ del macho 2.0 veces más largo que ancho y $0.71-0.73$ veces el antenómero VI.

\section{Nakaharathrips n.gen.}

Especie tipo: Anaphothrips sudanensis, Trybom 1911:1

English diagnosis: Antennae with 8 segments without division of the VI antennal segment.

Diagnosis: Antena con ocho antenómeros (Fig 7C), nunca presenta división parcial ni total del antenómero VI. Sensoria bifurcados en los segmentos III-IV de la antena, segmento VII pedicelado en la base. Cabeza ligeramente proyectada entre los ojos. Algunas veces con tubérculos anteromediales en la cabeza. Tres pares de setas ocelares, tres a cuatro pares de setas posoculares, alineadas posteriormente al ojo. Palpos maxilares trisegmentados. Sin setas mayores del pronoto. Espínula mesotorácica presente, espínula metatorácica ausente. Macrópteros. Cilios ondulados. Escama con cinco setas. Setas medias de los tergitos abdominales muy separadas. Tergito VIII con peine posteromarginal completo.

Comentario: El género Anaphothrips constituido por especies con 8 antenómeros como con 9 antenómeros. Al analizar la filogenia hallamos que el género Anaphothrips se divide en dos grandes grupos el de las especies con 8 antenómeros y las especies con 9 antenómeros, en cada caso hay un escenario evolutivo diferente. Al determinar cual es la tendencia evolutiva se observa que la presencia de 8 antenómeros es la condición plesiomórfica y la aparición de 9 segmentos por subdivisión del segmento VI es la apomorfía. Sabemos que según las reglas de clasificación cladística no deben mantenerse grupos con carcteres en estado plesiomórfico y apomórficco, porque obviamente va en contra de la novedad evolutiva hallada. Por esto el género debe dividirse en el género Anaphothrips para el grupo de especies con la condición apomórfica del caracter y el nuevo género Nakaharathrips para el grupo de especies con el caracter en estado plesiomórfico. Debe aclararse que algunas especies no presentan la total división del segmento VI, y esta se manifiesta en forma parcial por la presencia de una sutura incompleta que divide el antenómero VI en dos segmentos. Este estado de parcial división denota la tendencia a la novedad evolutiva por lo que estas especies se mantienen dentro de Anaphothrips. Los estudios morfológicos y topográficos de los segmentos VI y VII de la antena con nueve segmentos, demuestran que externamente la posición de las setas indica que se trata de una división del segmento VI, convirtiendo al segmento VII en un subsegmento del VI.

Revisión taxonómica: Para el presente trabajo se establece el cambio de estatus taxonómico para las especies registradas de Norte América (Nakahara 1995) y la única especie asiática de la que se dispuso, floralis. Sin embargo, no hay razón para suponer que las demás especies con 8 antenómeros no cumplan con la misma condición plesiomórfica que las ubique dentro de el nuevo género Nakaharathrips.

Nakaharathrips catawba n.comb.

Anaphothrips catawba Hood 1938a:348

Anaphothrips (Neophysopus) catawba:

Stannard 1968:277; Beshear 1979:210

Especie restringida a los EEUU (ha sido recolectada en siete estados). Sus hospederos son: Andropogon glomeratus, A. virginicus, Andropogon sp., Schizachyrium scoparium (=Andropogon scoparius), zacates, zacates anegados, muestras de suelo.

Nakaharathrips floralis n.comb.

Anaphothrips floralis Karny 1922:109

Especie endémica de Vietnam, conocida solamente para la localidad tipo (Saigón). Sus hospederos son: Clitoria ternatea (flores). 
Nakaharathrips helvolus n.comb.

\section{Anaphothrips helvolus}

Nakahara 1995:228

Especie restringida a los EEUU (ha sido recolectada en tres estados). Sus hospederos son: Panicum agrostoides, Trticum aestivum, Zea mays, Setaria sp., zacates.

\section{Nakaharathrips mexicanus n.comb.}

Anaphothrips mexicanus

Nakahara 1995:231

Especie endémica de México, recolectada en Xochimilco. Sus hospederos son: Bromus catharticus, Majorana sp.

\section{Nakaharathrips sudanensis n.comb.}

Anaphothrips sudanensis Trybom 1911:1, Mound 1963:21, Jacot-Guillarmod 1974:572, Bhatti 1978:87, Nakahara 1995:235, Mound \& Marullo 1996:91.

Especie cosmopolita, se le ha rerecolectado en el Nuevo Mundo, África, Asia y Australia. Es ampliamente polífaga, pero con preferencia por los zacates.

\section{Nakaharathrips trimaculatus n.comb.}

\section{Anaphothrips trimaculatus}

Nakahara 1995:237

Especie endémica de los EEUU, recolectada solamente en la Florida. Sus hopederos son: zacates, Panicum hemitomon.

\section{Gonzalezya gen. n.}

Especie tipo: Gonzalezya marinae n.sp., por designación original (Fig. 8A-B)

English diagnosis: Forewing with row of setae in vein I complete, posteroanagular setae well developed.

Coloración traslúcida sombreada con amarillo. Antenna 8-antenómeros (Fig. 7C), conos sensoriales bifurcados en los antenómeros III y IV; antenómero 1 sin seta dorsoapical; antenómeros III-VI con microtrichia, pedicelo modificado con forma de rótula circular y ligeramente asimétrico (Fig. 8B). Cabeza con 2 pares de setas ocelares; palpos maxilares con 4 segmentos. Ojos compuestos sin ommatidia de colores. Pronoto con 2 pares de setas pa bien desarrolladas (Fig. 8A), superficie dorsal con esculturación muy débil, basantra membranosa. Mesonoto con setas mediales naciendo en línea con las setas posterolaterales. Espínula (=tégula) mesotorácica presente. Metanoto con setas medias retrasadas lejanas al borde anterior. Espínula metatorácica ausente. Tarso bisegmentado. Ala 1 con cilios marginales ondulados; vena 1 con 9 setas discontinuas pero muy espaciadas entre sí con la siguiente distribución 3-3-1-1-1, vena 2 con hilera continua de 4 setas; escama con 5 setas de la vena y 2 setas discales. Ala II sin venación. Tergitos II-VIII con craspedum ancho y continuo en el margen posterior y reducido en el tergito 1 , VIII sin peine posteromarginal, setas B1-B2 en IX diferentes entre sí, nunca subiguales, $\mathrm{X}$ con corte longitudinal dorsal, pleurotergitos reducidos. Esternitos II-VII con craspedum marginal grande e interrumpido medialmente en el VII, setas discales ausentes, pleuroesternitos reducidos.
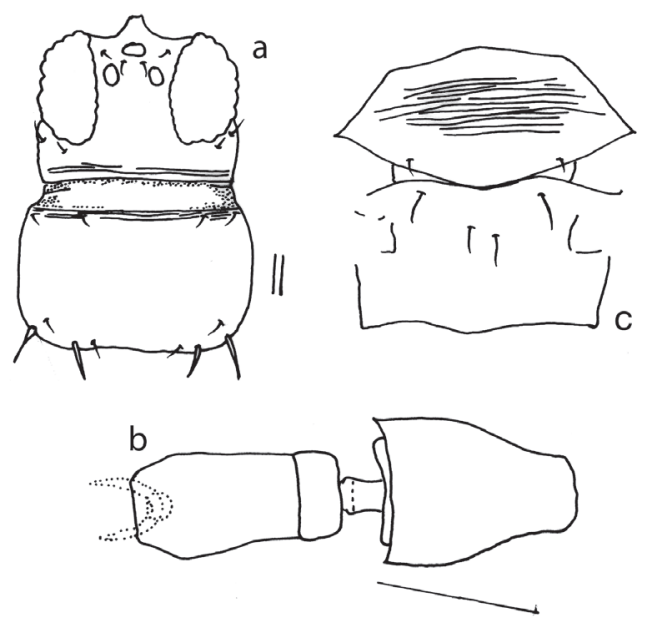

Fig. 8. Gonzalezya n.gen. marinae n.sp. A. Pronoto y cabeza. B. Detalle de la antena. C. Pterotórax. 


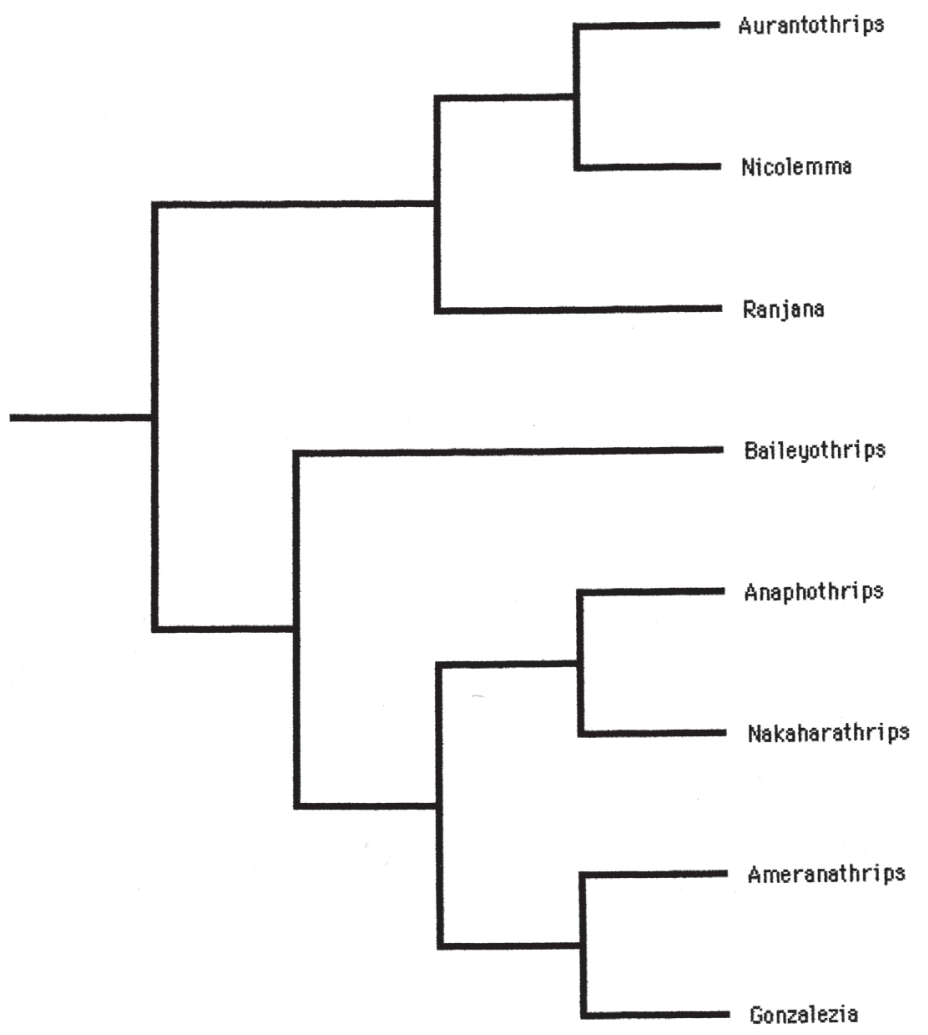

Fig. 9. Cladograma de los géneros del grupo genérico Anaphothrips neotropical; ci=0.75, ri=0.7, L= 41 (Apéndice 1).

Etimología: Este género a sido dedicado como reconocimiento por la labor de Yamileth González, Rectora de la Universidad de Costa Rica.

Comentario: Este grupo no es fácil de definir debido a que algunos caracteres de difícil polarización se comparten con otros géneros, por lo que existen dos alternativas: a) se trata de caracteres homoplásicos o b) son caracteres homólogos y los géneros que los exhiben pertenecen al mismo linaje. Los análisis filogenéticos efectuados indican que el nuevo género Gonzalezya pertenece al grupo Anaphothrips, se trata de uno de los táxones más deivados junto con Ameranathrips. Los caracteres morfológicos evidencian que estos dos géneros comparten importantes caracteres como a) reducción de los pleuritos, b) roseta accesorias en los espiráculos abdominales y c) una espermateca membranosa menos desarrollada que la presente en Nicolemma.

Gonzalezya marinae sp.n. (Fig. 8A-B)

English diagnosis: Forewing with irregular first row, with setae 9 very separated but with similar distances in the following distribution: 3-3-1-1-1; second vein with a row of 4 setae.

Material: Hembra macróptera (Holotipo) especímenes, rerecolectados en San José, Costa Rica en hojas de Anthurium. Además de seis especímenes (paratipos) con idénticos datos que el holotipo.

Descripción: Holotipo hembra macróptera. Cuerpo traslúcido sombreado con amarillo, margen externo de la coxa 1 café claro, metanoto traslúcido con sombra amarilla, antenómero 1 
mayormente traslúcido, II sombreado con amarillo, III-VI sombreado con café en el ápice, VIIVIII café, ala 1 fuertemente sombreada con café incluyendo la tégula, traslúcida en el tercio basal y en el ápice. Sin setas ocelares 1 en la parte anterior del triángulo ocelar, seta io III en posición 2, antenómeros III-IV con delicados conos sensoriales bifurcados. Ala 1 con la primera vena con una hilera irregular de 9 setas muy espaciadas entre sí con la siguiente distribución 3-3-1-1-1, vena dos con una hilera de 4 setas. Ala II sin venación. Tergito 1 totalmente esculturado, II-VI con eculturación medial, VIII esculturado con líneas transversales, setas B1-B2 en el IX tergito subiguales.

Medidas (en $\mu \mathrm{m}$ ): Holotipo hembra: Longitud corporal 920. Antenómeros $\mathrm{I}=22.5$ $\mathrm{II}=30 \mathrm{III}=45 \mathrm{IV}=37.5 \mathrm{~V}=40 \mathrm{VI}=42.5 \mathrm{VII}=$ $10 \mathrm{VIII}=20$. Pedicelo del antenómero $\mathrm{III}=7.5$. Collar del antenómero III= 17.5. Diámetro antero-posterior del pronoto $=95$. Diámetro transversal del pronoto $=162.5$. Longitud de las setas pa externas $=62.5$. Longitud de las setas pa inteiores $=62.5$. Diámetro antero-posterior del huevo $($ en oviducto $)=180$

Etimología: dedicada a Marina Salazar Mora.

\section{AGRADECIMIENTOS}

Agradezco a Gerardo Sánchez M. por los dibujos.

\section{RESUMEN}

Se presentan una revisión taxonómica y un análisis filogenético del grupo genérico Anaphothrips. En América Central hay algunos géneros cercanos a este género. Con base en el análisis de caracteres y su posible evolución, se segrega el nuevo género Nakaharathrips de Anaphothrips, y se cambia el estatus taxonómico de las subespecies de Aurantothrips al nivel de especie. El análisis filogenético muestra relación entre los géneros Anaphothrips, Ranjana,
Nakaharathrips n.gen., Aurantothrips, Nicolemma n.gen., Ameranathrips, Baileyothrips and Gonzalezya n.gen., donde el primer linaje está conformado por los géneros (Ranjana (Aurantothrips +Nicolemma)) y el segundo linaje por los géneros [(Anaphothrips + Nakaharathrips)(Baileyot hrips(Ameranathrips + Gonzalezya)].

Palabras clave: grupo Anaphothrips, nuevo estatus, filogenética, género nuevo, evolución de caracteres.

\section{REFERENCIAS}

Bhatti, J.S. 1978. A Revision of Karny's species of Anaphothrips of the Oriental Region (Thysanoptera: Thripidae). Oriental Insects 12: 1-27.

Brooks, D.R. \& E.O. Wiley. 1986. Evolution as Entropy: Toward a Unified Theory of Biology. Univ. of Chicago. Chicago, Illinois, EEUU. 335 p.

Jacot-Guillarmod, C.F. 1974. Catalogue of the Thysanoptera of the world, Part 3. Ann. Cape Prov. Mus. Nat. Hist. 7: 517-97.

Mound, L.A. \& R. Marullo. 1996. The thrips of Central and South America: An Introduction (Insecta: Thysanoptera). Mem. Entomol. 487 p.

Mound, L.A., B.S. Heming \& J.M. Palmer. 1980. Phylogenetic relationships between the families of recent Thysanoptera (Insecta). Zool. J. Linn. Soc. 69: 111-141.

Nakahara, S. 1995. Review of the Nearctic species of Anaphothrips (Thysanoptera: Thripidae). Insecta Mundi 9: 221-248.

Palmer, J.M. \& L.A. Mound. 1985. New World Thripidae (Thysanoptera) with nine-segmented antennae. Zool. J. Linn. Soc. 84: 181-194.

Retana-Salazar, A. \& S. Retana-Salazar. 2004. Hacia una lógica simple en la determinación de grupos biológicos: la especie y los grupos supraespecíficos (Forum). Rev. Biol. Trop. 52: 19-26.

Sakimura, K. 1967. Redescriptions of Anaphothrips orchidaceus and A. orchidearum (Thysanoptera: Thripidae). Fla. Ent. 50: 89-97. 


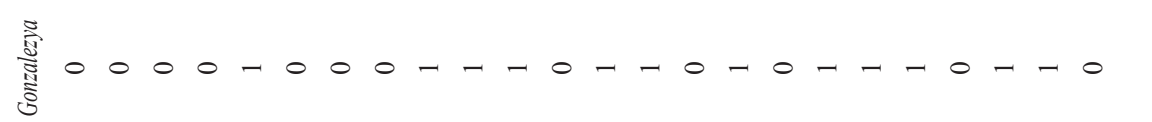

歖 $000-000-0-00--1-0--1-0-10$

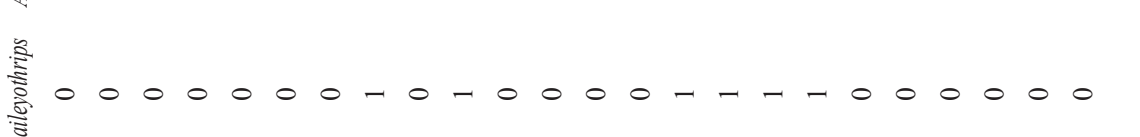

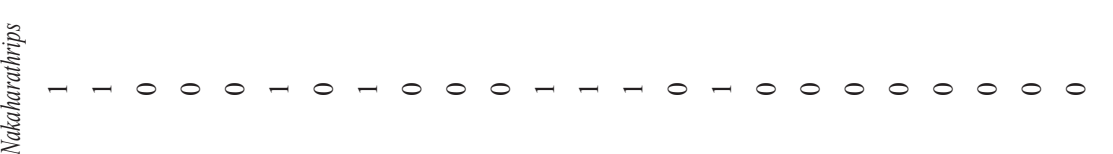

言 $00000-0-000--1-0-00000000$

竎

s.

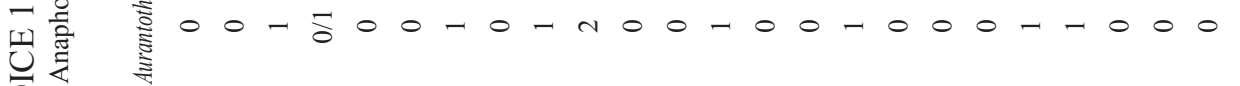

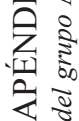

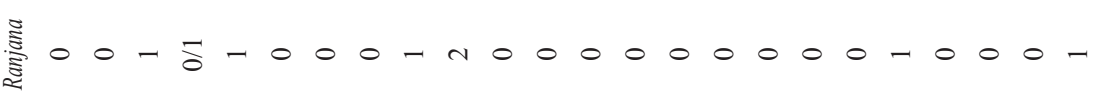

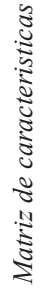

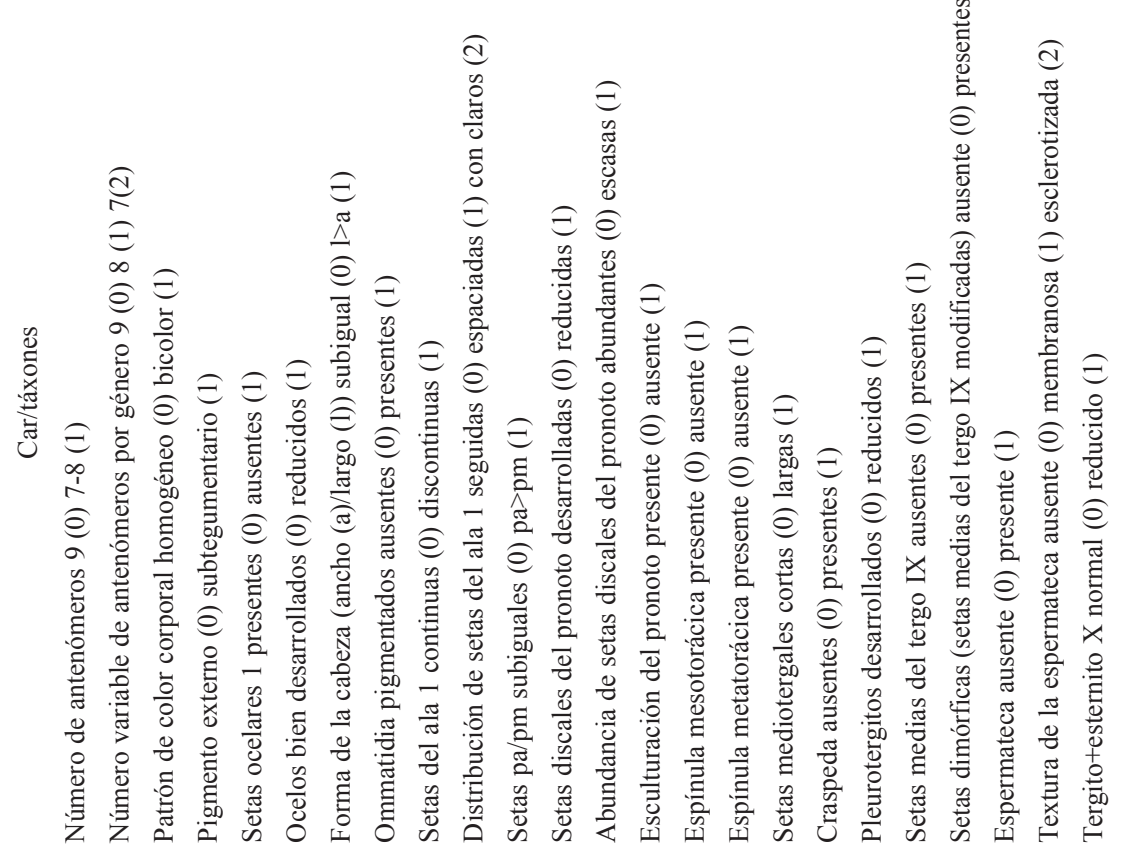

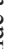


